\title{
ESTUDIO PARA EL ESTABLECIMIENTO DE UNA PLANTA DE RECUPERACIÓN Y TRATAMIENTO DE DESECHOS SÓLIDOS URBANOS EN EL MUNICIPIO DE CHITRÉ, PROVINCIA DE HERRERA, REPÚBLICA DE PANAMÁ
}

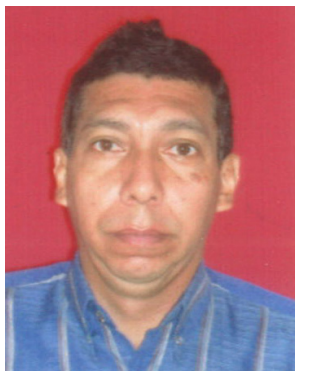

Fecha de recepción: 06/10/2018

\section{Osvaldo A. Solís A. ${ }^{1}$}

Universidad Metropolitana de Educación, Ciencia y Tecnología;

Panamá

osolis1959@hotmail.com

\section{RESUMEN}

La mayoría de las ciudades del mundo, confrontan problemas con sus vertederos municipales, relacionados con un manejo deficiente, ocasionado contaminación de los recursos naturales. En la República de Panamá, los vertederos están generando efectos perjudiciales sobre las comunidades y el ambiente; presentando una gestión, inadecuada, de la basura, que debe ser mejorada, urgentemente. En la ciudad de Chitré, el vertedero causa impactos ambientales, y riesgos a la salud de las personas, por lo cual, es urgente, buscar alternativas que aseguren mitigar los efectos que produce sobre la naturaleza y los habitantes del Distrito de Chitré. El objetivo general de la investigación es establecer una planta de recuperación y tratamiento de desechos sólidos urbanos, con el fin de evitar y reducir la contaminación en la ciudad de Chitré. La metodología incluyó una encuesta, mediante un cuestionario, aplicada, a una barriada de la ciudad de Chitré, con el propósito de obtener información, sobre la necesidad de establecer una planta de recuperación y tratamiento de residuos sólidos urbanos en el vertedero del municipio de Chitré. Algunas conclusiones son las siguientes: gran parte de la población del municipio de Chitré, tienen conocimiento de los problemas que representa el vertedero para la comunidad y el ambiente; el total de los habitantes encuestados consideran indispensable, el reciclaje de los desechos sólidos urbanos; la gran mayoría de los habitantes encuestados están de acuerdo con la instalación de una planta de recuperación y tratamiento de residuos sólidos urbanos, en el municipio de Chitré. Las recomendaciones fueron las siguientes: es indispensable establecer un plan de educación ambiental, relacionado con los residuos sólidos urbanos, en la ciudad de Chitré; algunas opciones de manejo para el vertedero del municipio de Chitré, pueden incluir: una planta de recuperación y tratamiento de residuos sólidos urbanos; o iniciar los estudios correspondientes para buscar un sitio, adecuado, para su traslado; o campañas de educación ambiental y reciclaje, en escuelas y la comunidad.

\footnotetext{
${ }^{1}$ Maestría en Ecología y Conservación; docente investigador; Universidad Metropolitana de Educación, Ciencia y Tecnología; osolis1959@hotmail.com
} 
Palabras clave: residuo sólido urbano, planta de recuperación y tratamiento, vertedero, municipio, educación ambiental.

\section{STUDY FOR THE ESTABLISHMENT OF A PLANT OF RECOVERY AND TREATMENT OF SOLID URBAN WASTE IN THE MUNICIPALITY OF CHITRÉ, PROVINCE OF HERRERA, REPUBLIC OF PANAMA}

\section{SUMMARY}

Most cities in the world, face problems with their municipal landfills, related to poor management, caused pollution of natural resources. In the Republic of Panama, landfills are having harmful effects on communities and the environment; presenting an inadequate management of garbage, which must be improved, urgently. In the city of Chitré, the landfill causes environmental impacts, and risks to the health of people, so it is urgent to look for alternatives that ensure mitigate the effects it produces on the nature and the inhabitants of the District of Chitré. The general objective of the research is to establish a plant for recovery and treatment of urban solid waste, in order to prevent and reduce pollution in the city of Chitré. The methodology included a survey, through a questionnaire, applied to a neighborhood of the city of Chitré, with the purpose of obtaining information on the need to establish a recovery and treatment plant for solid urban waste in the landfill of the municipality of Chitré . Some conclusions are the following: a large part of the population of the municipality of Chitré, are aware of the problems that the landfill represents for the community and the environment; the total of the surveyed inhabitants consider indispensable, the recycling of urban solid waste; the great majority of the surveyed inhabitants agree with the installation of a plant for the recovery and treatment of urban solid waste, in the municipality of Chitré. The recommendations were the following: it is essential to establish an environmental education plan, related to urban solid waste, in the city of Chitré; Some management options for the landfill in the municipality of Chitré may include: a recovery and treatment plant for urban solid waste; or initiate the corresponding studies to find a suitable site for their transfer; or environmental education and recycling campaigns, in schools and the community.

Keywords: urban solid waste, recovery and treatment plant, landfill, municipality, environmental education.

\section{INTRODUCCIÓN}

La Ley define como residuos sólidos aquellas sustancias, productos o sub productos en estado sólido o semisólido de los que su generador dispone, o está obligado a disponer, para ser manejados a través de un sistema que incluya, según corresponda, las siguientes operaciones o procesos: (i) minimización de residuos; (ii) segregación en la fuente; (iii) reaprovechamiento; (iv) almacenamiento; (v) recolección; (vi) comercialización; (vii) transporte; (viii) tratamiento; (ix) transferencia; y, (x) disposición final (Cox, et al., s/f). 
Estudio para el establecimiento de una planta de recuperación y tratamiento de desechos sólidos urbanos en el municipio de Chitré, provincia de Herrera, República de Panamá

Osvaldo A. Solís A. (70:81)

Los residuos sólidos municipales (RSM), conocidos comúnmente como basura, desecho o residuo, están compuestos por residuos orgánicos (alimentos, excedentes de comida, etc.), cartón, papel, madera y en general materiales inorgánicos como vidrio, plástico y metales. Estos residuos provienen generalmente de actividades domésticas, servicios públicos, construcciones y establecimientos comerciales, así como de residuos industriales que no se deriven de sus procesos (Rondón, et al., 2016).

La clasificación de los residuos sólidos urbanos es la siguiente:

\section{Según su origen:}

Residuo domiciliario

Residuo comercial

Residuo de limpieza de espacios públicos

Residuo de establecimiento de atención de salud

Residuo industrial.

Residuo de las actividades de construcción

Residuo agropecuario

Residuo de instalaciones o actividades especiales

\section{Según su gestión}

Residuos de ámbito municipal

Residuos de ámbito no municipal

\section{Según su peligrosidad:}

Residuos peligrosos

Residuos no peligrosos (Programa de Política y Gestión Ambiental de la Sociedad Peruana de Derecho Ambiental, 2009). Los impactos negativos potenciales del mal manejo de los residuos en el medio ambiente son evidentes y bien conocidos. A continuación se listan los principales: (i) Atmosféricos: Calidad del aire por emisiones de metano y dióxido de carbono, y el impacto de éstos como gases invernadero; emisión de dioxinas y furanos producto de la quema no controlada en basurales (principal fuente de emisiones de estos compuestos orgánicos persistentes o COP) en América Latina, así como de sulfuro de hidrógeno y otros. (ii) Suelos y geomorfología: Alteración de las propiedades físicas, químicas y de fertilidad; contaminación por presencia de aceites, grasas, metales pesados y ácidos, entre otros residuos; activación del proceso erosivo y cambio de topografía, entre otros. (iii) Aguas superficiales y subterráneas: Afectación de la calidad del agua y alteración de las características hidráulicas. (iv) Bióticos: Alteración de la cantidad de biomasa, del tipo de vegetación y fauna (Terraza, 2009). 
Los vertederos se pueden categorizar en los siguientes tipos:

\section{Controlados}

Un Vertedero o depósito controlado es una instalación para almacenamiento de residuos en superficie o subterráneo por tiempo indefinido en condiciones de total seguridad para el medio ambiente, provisto de las medidas constructivas y de control oportunas para garantizar que no se produzca la contaminación del medio (García, s.f.).

\section{No controlados}

Uno de los factores que daña la salud de los habitantes de las ciudades es la gran cantidad de basura que se va acumulando en terrenos no aptos. Los basurales a cielo abierto suelen ser uno de los focos infecciosos de muchas de las enfermedades que contraen a diario las miles de personas que habitan en sus inmediaciones (Frers, 2009). Hasta la fecha, el Relleno Sanitario es la técnica que mejor se adapta a nuestra región para disponer de manera sanitaria las basuras, tanto desde el punto de vista técnico como económico (Jaramillo, 1991). Planta de Recuperación: objetivos. $\Delta$ Obtener la separación de Componentes Reciclables (papel, vidrio, aluminio y plástico), de los Residuos Peligrosos Domiciliarios (patogénicos, latas de pintura, pilas, solventes) y de la materia orgánica. $\Delta$ Gestionar la comercialización de Componentes Reciclables. $\boldsymbol{\Delta}$ Enviar Residuos Peligrosos Domiciliarios y materia orgánica a deposición final en adecuado Relleno Sanitario. Planta de Recuperación y Tratamiento: objetivo. A Obtener la separación de Componentes Reciclables (papel, vidrio, aluminio y plástico), de los Residuos Peligrosos Domiciliarios (patogénicos, latas de pintura, pilas, solventes). $\Delta$ Reciclar la materia orgánica como lombricompuesto para utilizar en forma de Fertilizante Orgánico en horticultura y/o forestación. $\Delta$ Gestionar la comercialización de Componentes Reciclables y Fertilizantes Orgánicos. A Enviar Residuos Peligrosos Domiciliarios a disposición final en adecuado Relleno Sanitario (Scudelati \& Asociados, $\mathrm{s} / \mathrm{f}$ ). El objetivo general fue proponer el diseño de una planta de recuperación y tratamiento de desechos sólidos urbanos, con el fin de evitar y reducir la contaminación en la ciudad de Chitré.

\section{MATERIALES Y MÉTODOS}

La investigación se llevó a cabo en el Distrito de Chitré, Provincia de Herrera, República de Panamá, con los siguientes límites: al norte con los Distritos de Santa María y Parita; al sur con la Provincia de Los Santos; al este con El Golfo de Panamá y al oeste con el Distrito de Pesé.

El diseño fue no experimental, del tipo transeccional; con un estudio explicativo. Se utilizó un muestreo probabilístico, empleando la técnica del muestreo aleatorio simple, mediante una tómbola; con este procedimiento se escogió la barriada Brisas de Los Guayacanes, la cual, constituyó la población, con un total de XX viviendas (N). Para obtener el tamaño de la muestra (n), se utilizó, la ecuación (1) de muestreo, de poblaciones finitas; el resultado fue de 45 hogares. 
Para llevar a cabo la encuesta, se recorrió toda la barrida, encuestando a los jefes de familia, de cada vivienda; la selección de las casas, se efectuó por la técnica del muestreo aleatorio simple, a través de una tómbola; a las personas, se les explicó los objetivos del estudio, y su importancia para el municipio de Chitré. La encuesta estuvo conformada por preguntas abiertas y cerradas, relacionadas con el manejo de la basura, en la ciudad de Chitré.

Para trabajar la información, las preguntas, de cada encuesta, se analizaron, individualmente; después, se obtuvieron totales, por pregunta, de cada encuesta; y posteriormente, se determinaron, los porcentajes, de cada pregunta, en base a los totales obtenidos. Los resultados indicaron que la mayoría de los habitantes encuestados están de acuerdo con la instalación de una planta de recuperación y tratamiento de residuos sólidos urbanos, en el municipio de Chitré, lo que sugiere evidencia, suficiente, para aceptar la hipótesis de investigación, y no aceptar la hipótesis nula. Las técnicas empleadas fueron la reunión, la revisión bibliográfica, la encuesta y el cuestionario

A continuación se presenta una muestra de resultados más relevantes:

\section{RESULTADOS}

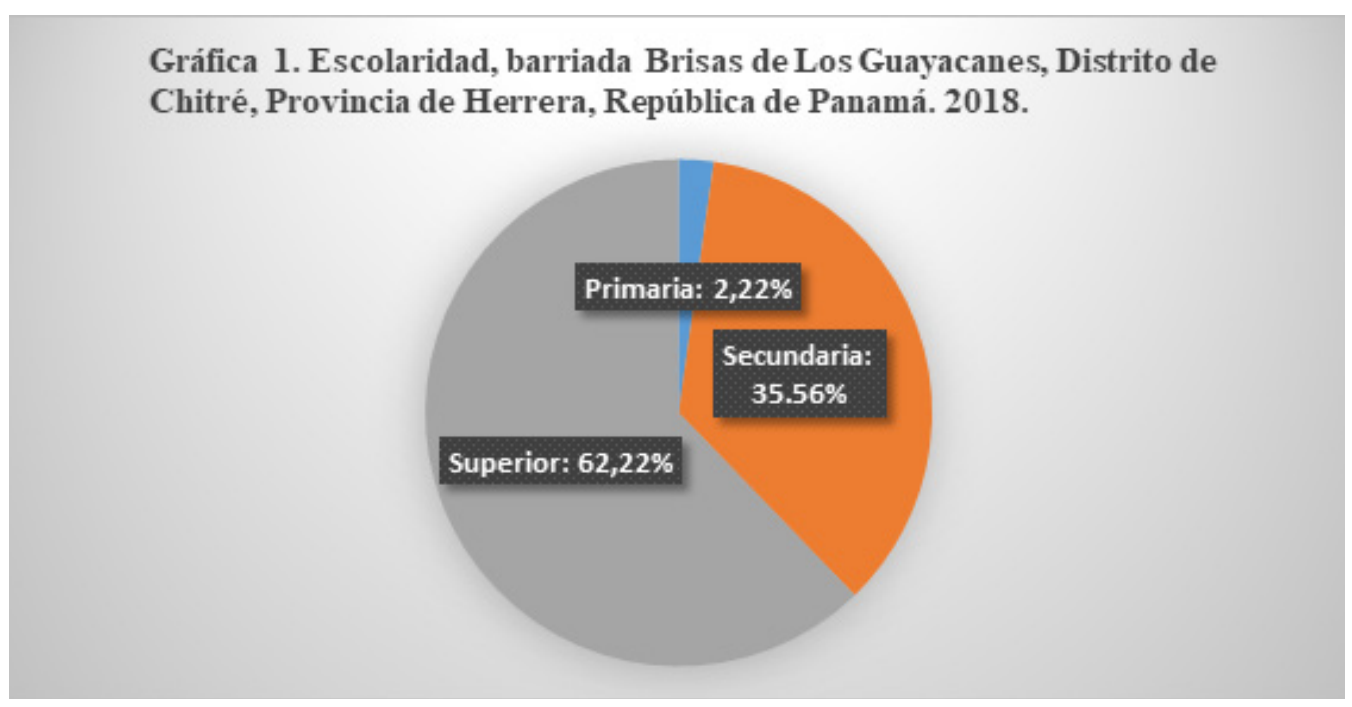

Fuente: Solis (2018)

La mayoria de las personas entrevistadas tienen una escolaridad superior y secundaria, indicando un nivel, adecuado, de comprensión y análisis de la situación del vertedero de la ciudad de Chitré (cuadro 1). 


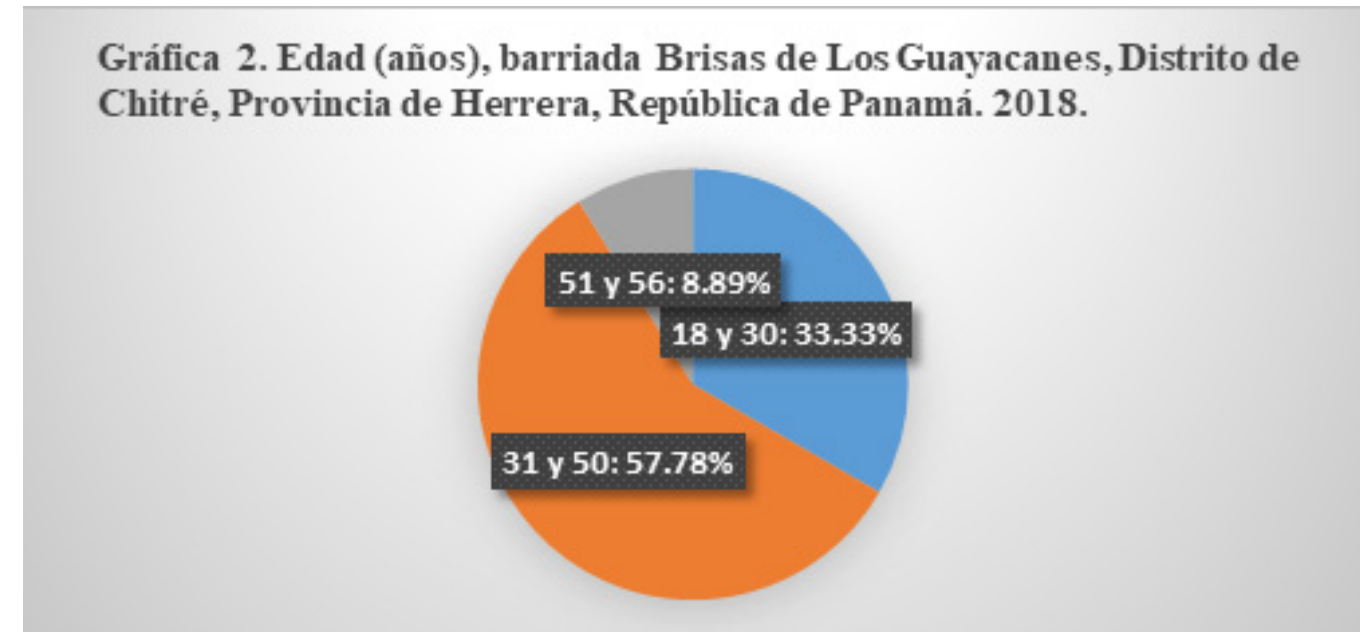

Fuente: Solis (2018)

Gran parte de los encuestados, presentaron edades entre los 31 y 50 años, señalando personas con juventud y adultez, es decir, con criterio formado (gráfica 2).

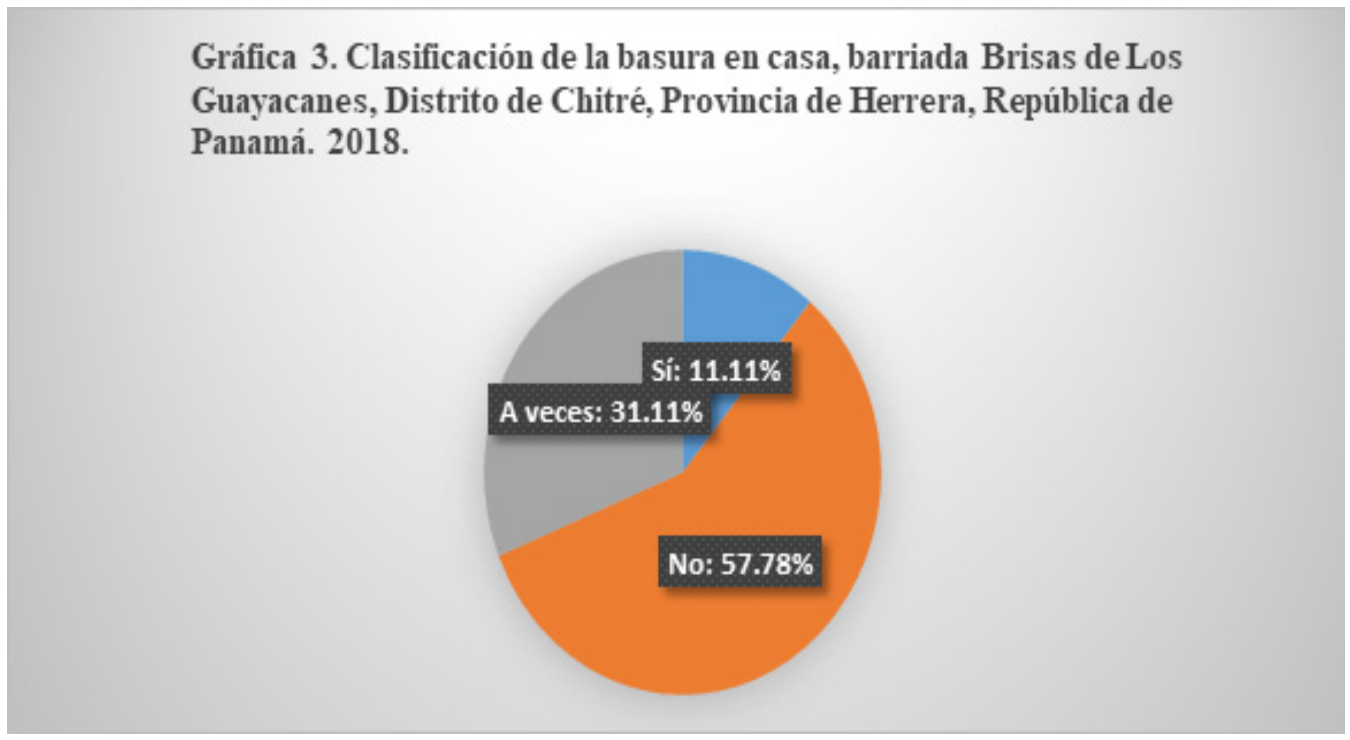

Fuente: Solis (2018)

La minoría de las personas clasifican la basura en sus viviendas, los demás no lo hacen, o en ocasiones, expresando un manejo, inadecuado, de los desechos sólidos urbanos, por falta de educación ambiental, en la mayoría de los encuestados (gráfica 3). 
Estudio para el establecimiento de una planta de recuperación y tratamiento de desechos sólidos urbanos en el municipio de Chitré, provincia de Herrera, República de Panamá

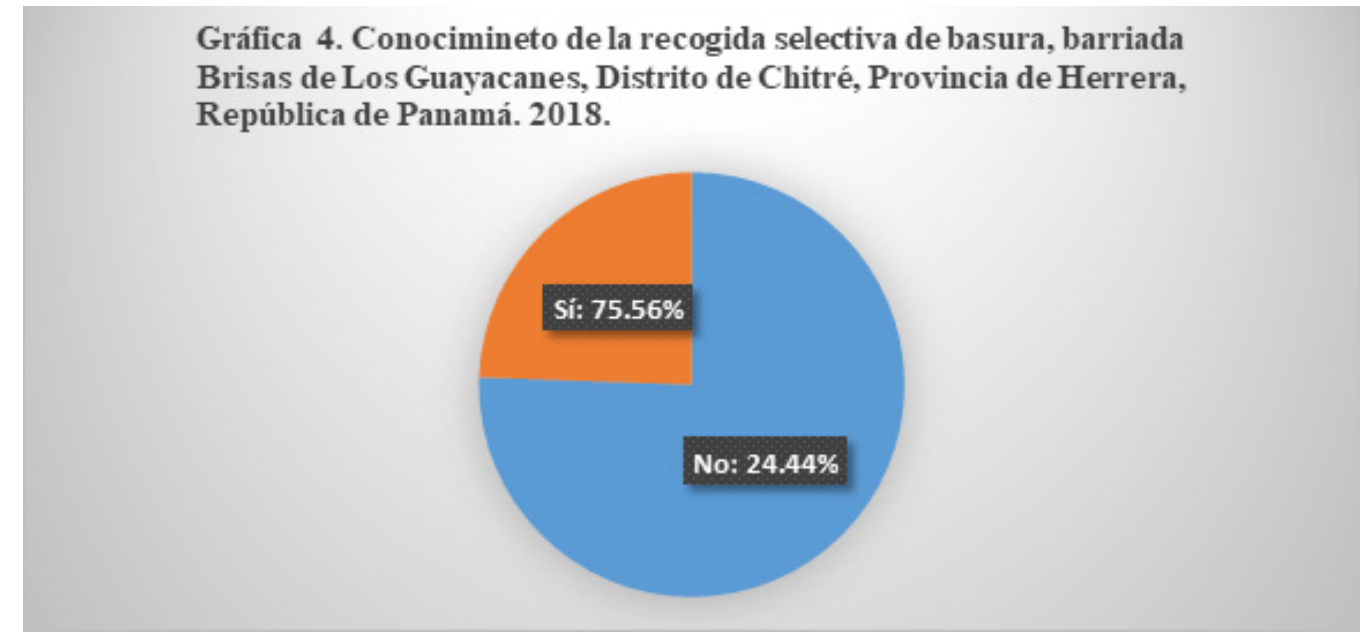

Fuente: Solis (2018)

Gran parte de los habitantes de la barriada, tienen información sobre la recogida selectiva, significando que tienen una idea acerca del reciclaje y su importancia, para evitar la contaminación que producen los vertederos (gráfica 4).

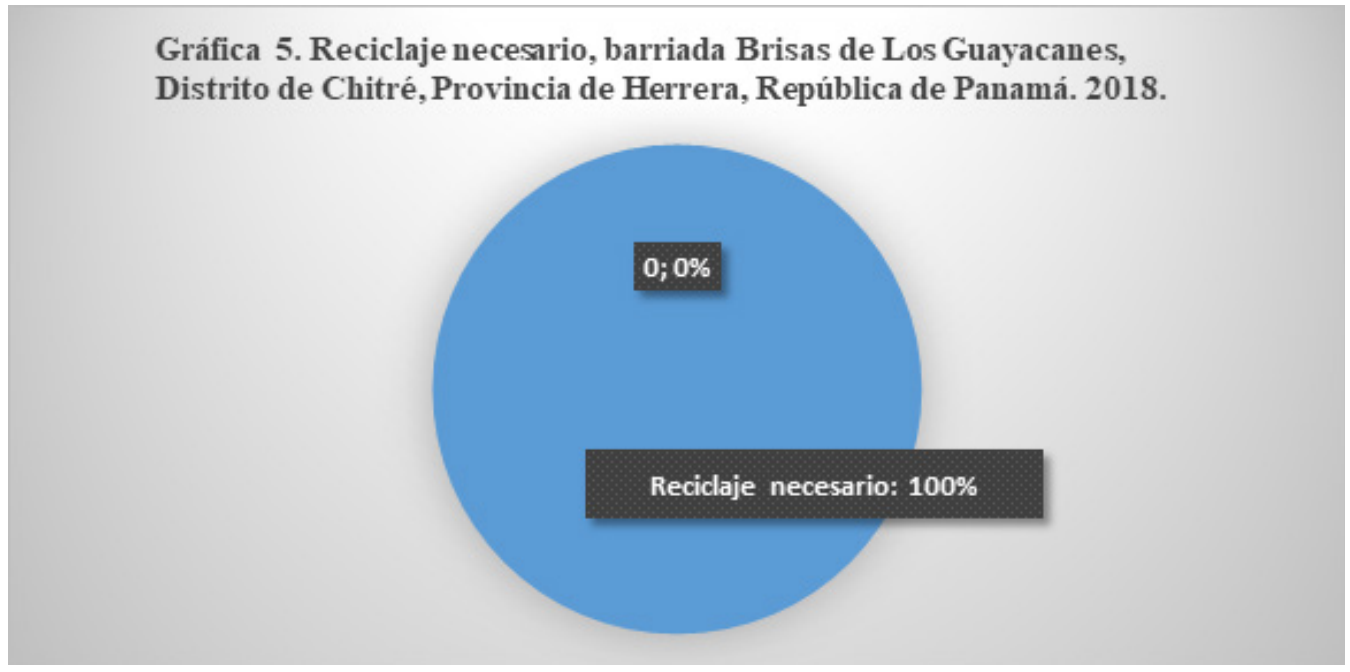

Fuente: Solis (2018)

E1 $100 \%$ de la población encuestada, considera necesario el reciclaje, por ser una solución a los problemas de salud y ambientales que causan los vertederos sin manejo eficiente (cuadro 5). 
Gráfica 6. Materiales de desecho más frecuentes en la basura, barriada Brisas de Los Guayacanes, Distrito de Chitré, Provincia de Herrera, República de Panamá. 2018.

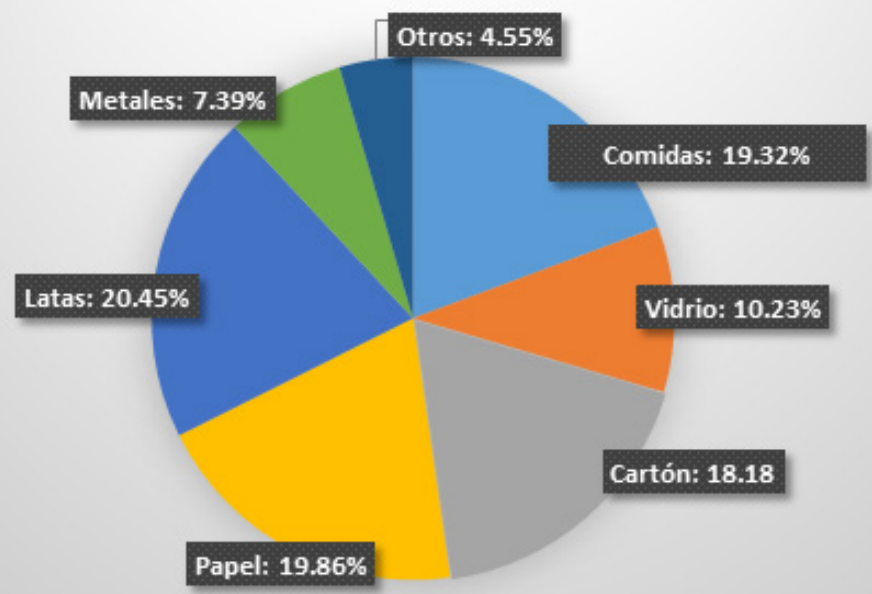

\section{Fuente: Solis (2018)}

Los materiales de desechos que son tirados en la basura, con más frecuencia, en orden descendente, son los siguientes: latas, comidas, papel y cartón; con frecuencia intermedia, el vidrio; y en menor cantidad: metales y otros; indicando, que las compras incluyen productos relacionados con éstos materiales (Gráfica 6).

Gráfica 7. Servicio de recolección de basura, barriada Brisas de Los Guayacanes, Distrito de Chitré, Provincia de Herrera, República de Panamá. 2018.

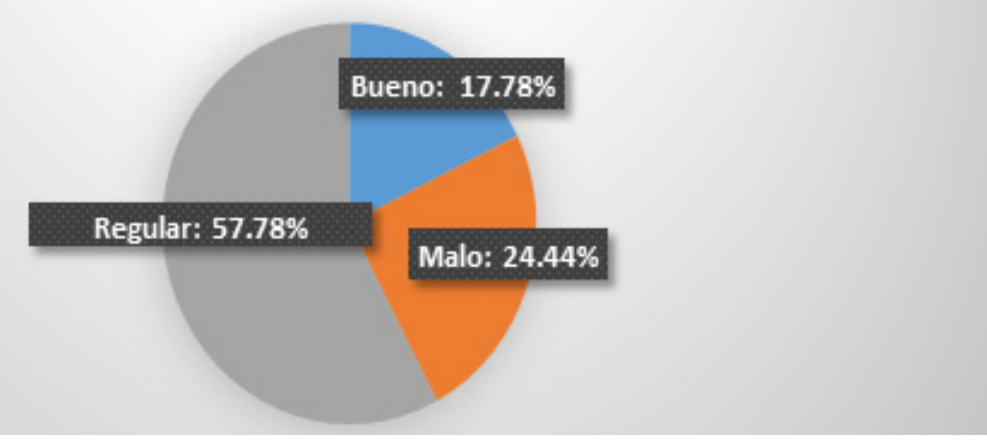

Fuente: Solis (2018)

La población de la barriada considera, al servicio de recolección de basura, público, regular en mayor porcentaje; y en menor, bueno; ocupando un grado intermedio, malo; señalando insatisfacción en la recogida de basura por el municipio de Chitré (gráfica 7). 
Estudio para el establecimiento de una planta de recuperación y tratamiento de desechos sólidos urbanos en el municipio de Chitré, provincia de Herrera, República de Panamá

Gráfica 8. Materiales para reciclar, barriada Brisas de Los Guayacanes, Distrito de Chitré,Provincia de Herrera, República de Panamá. 2018.

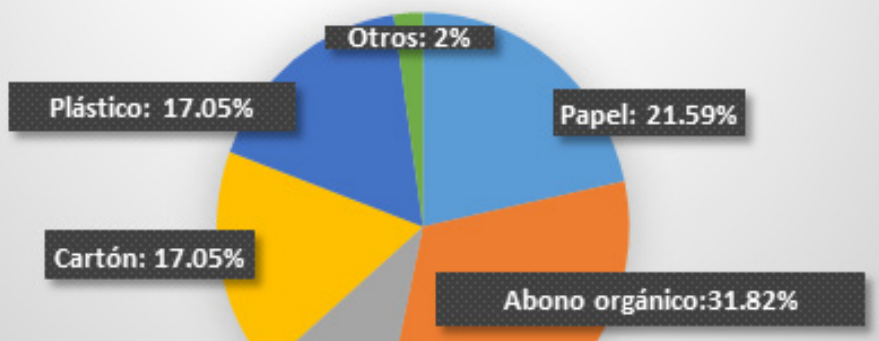

Vidrio: $10.23 \%$

Fuente: Solis (2018)

El material que prefieren las personas reciclar, en mayor cantidad, es el abono orgánico; seguido por el papel, el cartón y el plástico; con menores preferencias estuvo el vidrio y otros; debido a los beneficios del abono orgánico en el crecimiento de las plantas, y su fácil uso; el papel, cartón y plástico, vidrio y otros, requiere de recolección, almacenamiento, transporte y venta; este proceso implica trabajo, lo que impide, para algunas o muchas personas, llevarlo a cabo por limitaciones de tiempo o esfuerzo (gráfica 8).

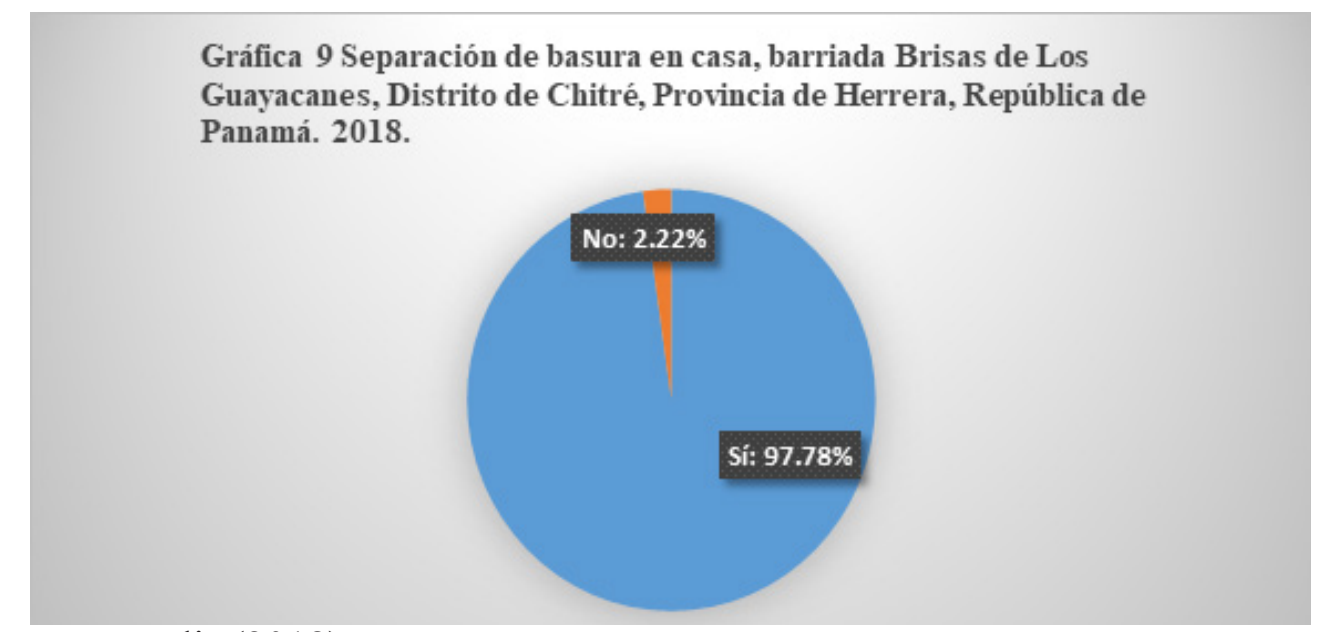

Fuente: Solis (2018)

Gran cantidad de la población, está dispuesta a separar la basura en sus viviendas; y muy poco, no; expresando el conocimiento y la importancia que las personas le dan a la separación de la basura en casa (gráfica 9). 


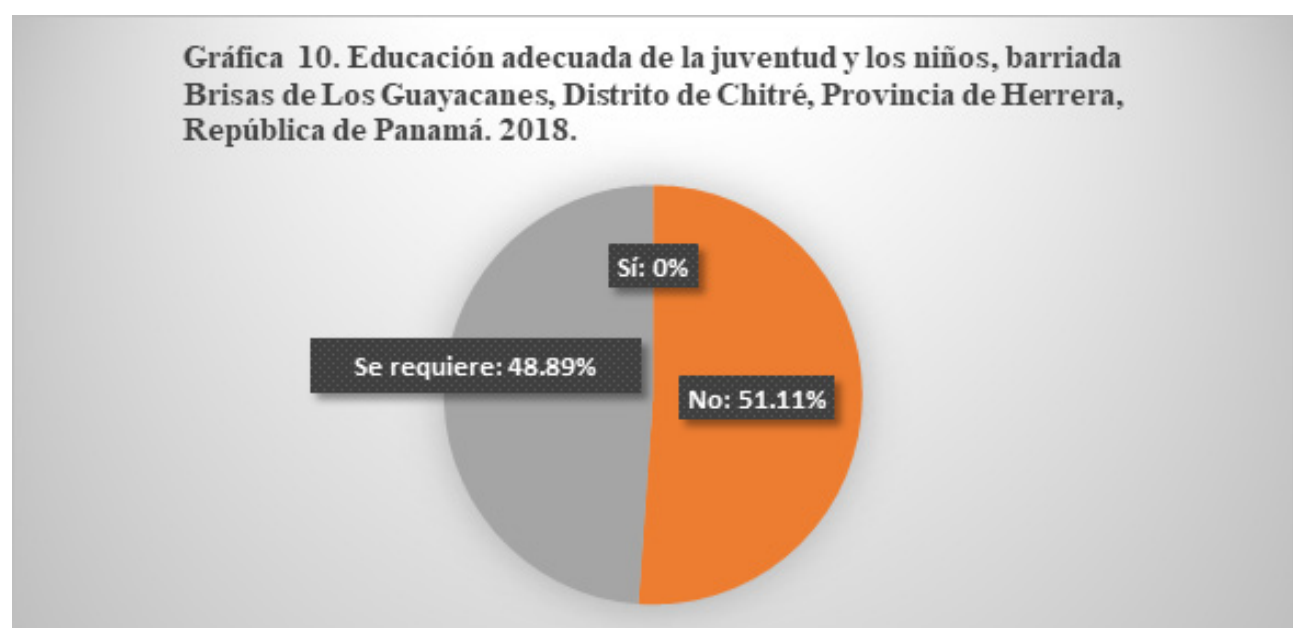

Fuente: Solis (2018)

Gran parte de la población encuestada, contestó, que no hay educación, adecuada, sobre el reciclaje, en la juventud y en los niños; seguido del grupo que dijo que se requiere una educación, adecuada; y no hubo respuesta en que hay una educación adecuada; debido a que muchas personas tienen una comprensiómn de la problemática de la basura en el municipio de Chitré (gráfica 10).

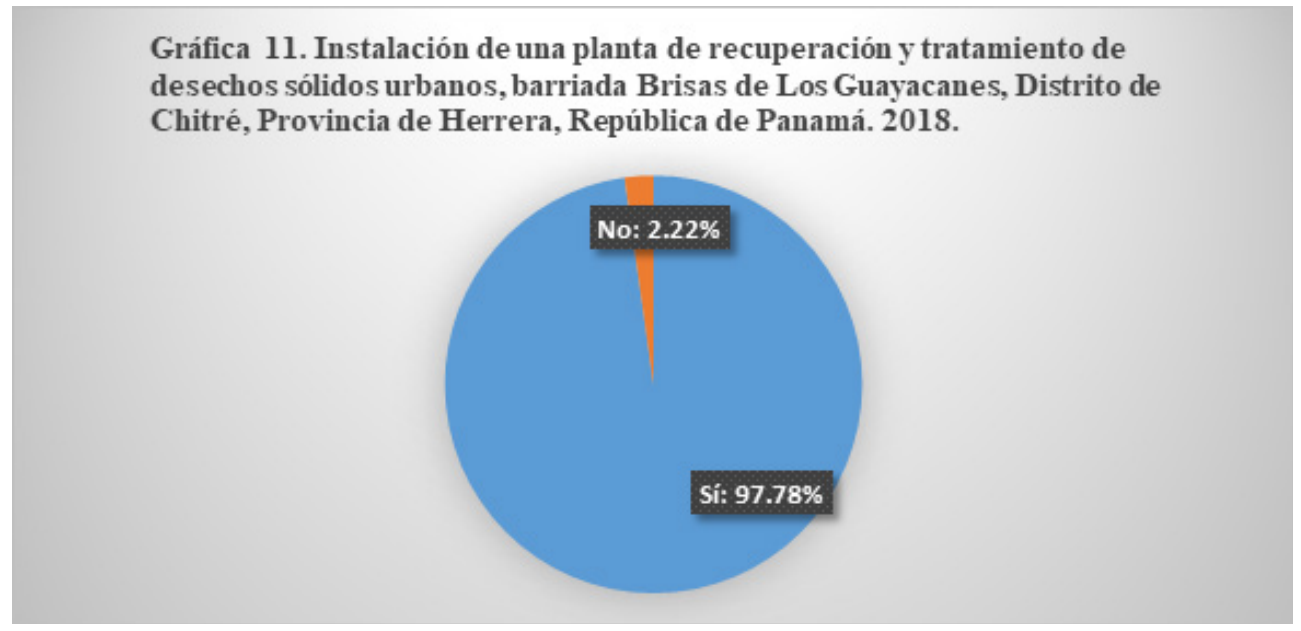

Fuente: Solis (2018)

La gran mayoría de los habitantes encuestados están de acuerdo con la instalación de una planta de recuperación y tratamiento de residuos sólidos urbanos, en el municipio de Chitré; mientras muy pocos no estan de acuerdo; lo que nos dice que la población tiene conocimiento del manejo, efeciente, de los residuos sólidos urbanos, por medio de una planta de recuperación y tratamiento; otros consideran que se debe trasladar el vertedero a otro sitio, más apto, y no instalar la planta (gráfica 11). 
Estudio para el establecimiento de una planta de recuperación y tratamiento de desechos sólidos urbanos en el municipio de Chitré, provincia de Herrera, República de Panamá

\section{CONCLUSIONES}

Gran parte de la población del municipio de Chitré, tiene conocimiento de los problemas que representa el vertedero para la comunidad y el ambiente.

Una minoría de los habitantes clasifica la basura en sus casas, y la mayoría no lo lleva a cabo.

Muchas personas tienen información sobre la recogida selectiva de basura.

El total de los habitantes encuestados consideran indispensable, el reciclaje de los desechos sólidos urbanos.

Los materiales de desechos con mayor frecuencia en la basura de las casas son los siguientes: latas, comidas, papel y cartón; seguido del vidrio; y en menor grado, metales y otros.

El servicio de recolección de basura, por el municipio de Chitré, es considerado, por la mayoría de los encuestados: regular; un grupo menor, dice que es malo; y muy pocos, que es bueno.

Gran cantidad de personas prefieren reciclar abonos orgánicos; otros pobladores, en menor cantidad: papel, cartón y plástico; y algunos: vidrio y otros.

La mayoría de los habitantes está dispuesta a separar, los residuos sólidos urbanos, en sus viviendas.

Gran parte de la población encuestada, contestó, que no hay educación, adecuada, sobre el reciclaje en la juventud y en los niños de la coumnidad.

La gran mayoría de los habitantes encuestados están de acuerdo con la instalación de una planta de recuperación y tratamiento de residuos sólidos urbanos, en el municipio de Chitré.

\section{RECOMENDACIONES}

Es indispensable establecer un plan de educación ambiental, relacionado con los residuos sólidos urbanos, en la ciudad de Chitré.

Algunas opciones de manejo para el vertedero del municipio de Chitré, pueden incluir: una planta de recuperación y tratamiento de residuos sólidos urbanos; o iniciar los estudios correspondientes para buscar un sitio, adecuado, para su traslado; o campañas de educación ambiental y reciclaje, en escuelas y la comunidad. 


\section{REFERENCIAS BIBLIOGRÁFICAS}

- Botamino García, J. I. (2007). DEPÓSITO DE RESIDUOS EN VERTEDERO. Madrid: Escuela de Organización Industrial (EOI). Obtenido de https://www.eoi. es/es/savia/publicaciones/20165/deposito-de-residuos-en-vertedero

- Cox Harman, C., Soto Torres, K., \& Velásquez Lucen, R. (2010). MANUAL TÉCNICO DE DIFUSIÓN MANEJO DE RESIDUOS SÓLIDOS PARA ALBERGUES EN ZONAS RURALES. Lima, Perú: Bachmann SCRL. Obtenido de https://www.mincetur.gob.pe/wp-content/uploads/documentos/turismo/ consultorias/turismo-TRC/Manual_Tecnico_Residuos_Solidos_Albergues_ Rurales_2010.pdf

- Frers, C. (Noviembre de 2009). Magazine online Waste. Obtenido de https://waste. ideal.es/vertederos.htm

- Jaramillo, J., \& Zepeda, F. (1991). Residuos sólidos municipales : guía para el diseño, construcción y operación de rellenos sanitarios manuales. Colombia: OPS.

- Programa de política y gestión ambiental de la sociedad peruana de derecho ambiental. (2009). Manual de Residuos Sólidos Urbanos. Perú: Lerma Gómez, EIRL. Obtenido de https://spda.org.pe/

- Rondón Toro, E., Szantó Narea, M., Pacheco, J., Contreras, E., \& Gálvez, A. (2016). Guía general para la gestión de residuos sólidos domiciliarios (Vol. 2). Santiago, Chile: ONU. Obtenido de https://repositorio.cepal.org/handle/11362/40407

- SCUDELATI \& ASOCIADOS. (S/f). Plantas de recuperación / Tratamiento de residuos urbanos. Bahía Blanca, Argentina: SCUDELATI \& ASOCIADOS. Obtenido de http://www.ecopuerto.com/Bicentenario/informes/ PLANTATRATAMIENTOSCUDEL.pdf

- Terraza, H. (2009). Manejo de Residuos Sólidos: Lineamientos para un Servicio Integral, Sustentable e Inclusivo. NOTA TECNICANo. IDB - TN - 101 : Banco Inter - Americano de Desarrollo. Obtenido de https://publications.iadb.org/publications/ spanish/document/Manejo-de-Residuos-S\%C3\%B3lidos-Lineamientos-para-unServicio-Integral-Sustentable-e-Inclusivo.pdf 